\title{
Comparison of the short-term efficacy between docetaxel plus carboplatin and 5-fluorouracil plus carboplatin in locoregionally advanced nasopharyngeal carcinoma
}

\author{
This article was published in the following Dove Press journal: \\ OncoTargets and Therapy \\ 18 August 2016 \\ Number of times this article has been viewed
}

\author{
Xing $\operatorname{Lv}^{1,2, *}$ \\ Wei-Xiong $\mathrm{Xia}^{1,2, *}$ \\ Liang-Ru Ke ${ }^{1,2}$ \\ Jing Yang ${ }^{1,2}$ \\ Wen-Zhe Qiu'1,2 \\ Ya-Hui Yu ${ }^{1,2}$ \\ Hu Liang ${ }^{1,2}$ \\ Xin-Jun Huang ${ }^{1,2}$ \\ Guo-Yin Liu',2 \\ Qi Zeng',2 \\ Xiang Guo ${ }^{1,2}$ \\ Yan-Qun Xiang ${ }^{1,2}$ \\ 'Key Laboratory of Oncology \\ in South China, ${ }^{2}$ Department of \\ Nasopharyngeal Carcinoma, Sun \\ Yat-sen University Cancer Center, \\ Guangzhou, People's Republic of \\ China
}

*These authors contributed equally to this work

Correspondence: Xiang Guo; Yan-Qun Xiang

Department of Nasopharyngeal Carcinoma, Sun Yat-sen University Cancer Center, 65I Dongfeng Road East, Guangzhou 510060, Guangdong, People's Republic of China

Tel +862087343359

Fax +862087343392

Email guoxiang@sysucc.org.cn; xiangyq@sysucc.org.cn

\begin{abstract}
Objective: Platinum-based chemotherapy in combination with radiotherapy is a standard treatment strategy for locoregionally advanced nasopharyngeal carcinoma (NPC). This study aimed to investigate the long-term efficacy and tolerability of inductive chemotherapy with docetaxel plus carboplatin (TC) or 5-fluorouracil plus carboplatin (FC) followed by concurrent radiation therapy in patients with NPC.
\end{abstract}

Methods: Patients $(\mathrm{N}=88)$ were randomized to receive TC or FC as inductive therapy followed by concurrent radiotherapy (60-70 Gy) with two cycles of carboplatin (area under the curve $=5 \mathrm{mg} \cdot \mathrm{h} / \mathrm{L}$ ). Patients were followed up for 8 years. Primary end point was progression-free survival (PFS). Secondary end points included overall survival (OS), toxicity, tumor response, distant metastasis-free survival, and local recurrence-free survival.

Results: At the end of the follow-up period, 31 patients died, 32 had disease progression, eleven had cancer recurrence, and 25 had distant metastasis. Overall, there was no difference between treatment groups with regard to response or survival. We found that following induction and concurrent chemoradiotherapy, the majority of patients showed a complete response ( $~ 96 \%-98 \%$ for induction therapy and $82 \%-84 \%$ for comprehensive therapy) to both therapies. PFS and OS were also similar between groups. The rate of PFS was $63.6 \%$ for both FC and TC and that of OS was $65.9 \%$ and $63.5 \%$, respectively. The overall incidence of grade $3-4$ adverse events in the TC group $(20.5 \%)$ was higher than in the FC group (10.7\%). Neutropenia and leukopenia were the most common grade 3-4 adverse events in the TC group, and mucositis was the most common in the FC group.

Conclusion: These data indicate that TC and FC therapies have similar efficacy in treating locally advanced NPC and both are well tolerated.

Keywords: nasopharyngeal cancer, carboplatin, 5-fluorouracil, docetaxel, tolerability

\section{Introduction}

Nasopharyngeal carcinoma (NPC) is the most common malignancy of the head and neck in southern part of the People's Republic of China. ${ }^{1,2}$ Approximately 21.4/100,000 persons per year will develop NPC in the People's Republic of China, which is higher than the worldwide rate of incidence $\left(<1 / 100,000\right.$ persons per year). ${ }^{1,2}$ The gold standard of NPC treatment is a combination of radiotherapy and chemotherapy, commonly involving 5-fluorouracil (5-FU) and cisplatin. ${ }^{3-6}$ Treatment failure for primary NPC is common, with failure rates of $7 \%-13 \%$ for residual tumor and $15 \%-58 \%$ for recurrent NPC. ${ }^{4,7}$ Treatment of recurrent or residual NPC is even more difficult with an average 5 -year survival rate of $20 \% .^{7}$ 
The use of induction chemotherapy followed by concurrent chemotherapy is of great interest. ${ }^{6}$ Several studies have found that adjuvant platinum-based chemotherapy followed by concurrent platinum-based chemoradiotherapy showed survival benefits in patients with NPC. ${ }^{8-10}$

Docetaxel is a chemotherapeutic that is widely used in therapy of head and neck cancer. ${ }^{11}$ The administration of docetaxel is simple, and duration of docetaxel therapy is short. One study found that the 2-year survival rate and disease-free survival rate in patients with locoregionally advanced NPC treated with docetaxel plus carboplatin (TC) for two courses of inductive therapy followed by concurrent cisplatin treatment and radiotherapy were $91.8 \%$ and $78.5 \%$, respectively. ${ }^{12}$ Although a number of studies have compared the efficacy and tolerability of cisplatin plus docetaxel with cisplatin plus 5-FU in NPC, only one short-term (1 year) study has compared the efficacy and tolerability of TC with that of 5-FU plus carboplatin (FC) as inductive therapy followed by concurrent chemoradiation therapy in treating locally advanced NPC..$^{13}$ The aim of this Phase II study was to compare the long-term efficacy and tolerability between FC and TC as inductive therapy followed by concurrent chemoradiation therapy in Chinese patients with locoregionally advanced NCP.

\section{Methods}

This prospective, parallel, open-label, randomized Phase II study compared the longterm clinical efficacy and safety of induction chemotherapy with TC (TC group) and FC (FC group) in patients with locally advanced NPC. The study was performed in accordance with the Declaration of Helsinki. The study and protocol were approved by the ethics committee of Sun Yat-sen University, and all patients gave their written informed consent.

\section{Study population}

Eligible patients were aged 18-60 years with pathologically diagnosed differentiated nonkeratinizing carcinoma or undifferentiated nonkeratinizing carcinoma (World Health Organization [WHO] staging system) of stage T3-4N0-3M0 (UICC2002 staging system). Included patients had not received initial therapy, chemotherapy, surgical intervention, or radiotherapy for their disease prior to study enrollment. Patients had to have a favorable overall health, no contraindications for chemotherapy, a Karnofsky score $\geq 80$, and a performance score $\leq 2$. Included patients had normal hematological values: absolute neutrophil count was $\geq 2 \times 10^{9} / \mathrm{L}$, platelet count was $\geq 100 \times 10^{9} / \mathrm{L}$, and hemoglobin was $\geq 9 \mathrm{~g} / \mathrm{dL}$; total bilirubin was greater than the upper limit of normal
(ULN); and aspartate transaminase and/or alanine transaminase was $>1.5$ times the ULN and alkaline phosphatase was $>2.5$ times the ULN.

Patients were excluded if the pathological findings indicated that they had type I NPC (WHO staging system) or adenocarcinoma, serious liver or kidney dysfunction, or heart disease. Patients with metastasis before therapy and those with other diseases or conditions that may impact the study results were also excluded.

Patients were withdrawn from the study at the discretion of the clinician if there was evidence of disease progression and for being noncompliant. Patients were also allowed to withdraw from the study for participating in another trial, receiving other chemotherapeutics, a need to undergo surgery, or if they were intolerant to the chemotherapy.

\section{Treatment regimens}

In TC group, patients were treated with docetaxel $\left(70 \mathrm{mg} / \mathrm{m}^{2}\right)$ plus carboplatin (area under the curve $=5 \mathrm{mg} \cdot \mathrm{h} / \mathrm{L}$ ) for two courses ( 3 weeks per course) followed by two courses (3 weeks per course) of carboplatin (area under the curve $=5 \mathrm{mg} \cdot \mathrm{h} / \mathrm{L}$ ) alone. Patients received concomitant radiotherapy during chemotherapy. Radiotherapy was conducted with cobalt 60 or photon beams (6 or $8 \mathrm{MV}$ ) of linear accelerator five times weekly (2.0 Gy per irradiation) for 6-9 weeks. The total dose of irradiation was $60-78$ Gy administered at the nasopharynx and 60-70 Gy for radical therapy of the neck lymph nodes. Determination of the target volume for radiation and location of prophylactic irradiation are described in Table S1.

In FC group, patients were administered 5-FU (120-hour intravenous infusion of $4 \mathrm{~g} / \mathrm{m}^{2}$ as one cycle and stopped till the third week, and then next cycle was started) plus carboplatin. Treatment otherwise was the same as the TC group. Oral dexamethasone $(7.5 \mathrm{mg}$ ) was given twice daily before, during, and after chemotherapy with docetaxel to prevent drug-induced retention of water and sodium.

Dose adjustment of chemotherapeutics was based on the National Cancer Institute Common Terminology Criteria for Adverse Events (CTCAE) 3.0 (Table S2).

\section{Concomitant therapies}

One hour before chemotherapy, a 5-HT3 antagonist was used for antiemetic therapy followed within 5-15 minutes by oral or intravenous administration of dexamethasone (15-20 mg). Starting on the second day of chemotherapy, dexamethasone ( $7.5 \mathrm{mg}$ ) and metoclopramide ( $20 \mathrm{mg}$; or a 5-HT3 antagonist) were administered twice daily for 3 days. 
Application of granulocyte colony-stimulating factor (G-CSF) was not recommended unless the white blood cell count or absolute neutrophil count dropped. If neutropenia of grade 3-4, as defined by CTCAE, or fever due to neutropenia (ie, oral temperature $\geq 38.3^{\circ} \mathrm{C}$ [axillary temperature $\geq 37.8^{\circ} \mathrm{C}$ ] or oral temperature $\geq 38^{\circ} \mathrm{C}$ [axillary temperature $\geq 37.5^{\circ} \mathrm{C}$ ] for longer than 1 hour) occurred, secondary treatment with G-CSF was given at the discretion of the physician. In addition, the dosage of the chemotherapeutic was adjusted. G-CSF therapy was administered $\geq 24$ hours following completion of chemotherapy.

Supportive therapy, such as pain medication, blood transfusion, and intravenous nutrition, was administered as necessary. Patients were not allowed to receive treatment with other drugs or undergo other radiotherapy/chemotherapy procedure during the study. However, other antitumor therapies were used if the patients showed signs of disease progression with study treatment.

\section{Assessment of efficacy and tolerability}

Physical examination, nasopharyngeal fiberscopy, and magnetic resonance imaging were employed to evaluate the therapeutic efficacy for lifelong follow-up. The evaluations of the therapeutic efficacy started at 1 and 3 months after chemotherapy. Then, they were done once every 3 months till the end of third year after chemotherapy, twice a year till the end of the fifth year after chemotherapy, and once a year from the sixth year after chemotherapy. According to the WHO Response Evaluation Criteria in Solid Tumors (2003), the therapeutic efficacy was classified as complete remission (CR), partial remission (PR), stable disease (SD), and progressive disease. Progression-free survival (PFS) and overall survival (OS) were evaluated at $1,2,3,5$, and up to 8 years.

Safety was assessed throughout the study using CTCAE 3.0.

\section{Statistical analysis}

A sample size of 44 patients per group was necessary based on our clinical research experience of a 5-year PFS rate of $70 \%$ in patients with late-stage nasopharyngeal cancer and who were receiving combined radiotherapy and chemotherapy for local-regional treatment. The power calculations used a group difference of 0.1 , a type I error of 0.05 , a power of 0.8 , a 3-year enrollment period, a 5-year follow-up duration, and a dropout rate of $8 \%$.

All the patients meeting the inclusion criteria were randomly assigned to the TC group or FC group at a ratio of 1:1. Dynamic randomization (Pocock and Simon minimization method) was employed to randomize patients. ${ }^{13}$ The randomization in this study was conducted in a concentrated manner, and randomization was performed centrally.

The primary end point of this trial was PFS. Secondary end points included OS, toxicity, tumor response, distant metastasis-free survival (DMFS), and local recurrence-free survival (LRFS). Duration of event (ie, PFS, OS, DMFS, LRFS) was calculated from the date of randomization to the date of event occurring or censoring.

The intention-to-treat population included all patients randomly assigned. The safety population included all randomized patients who completed radical radiotherapy. Kaplan-Meier curves were generated to estimate the rates of PFS, OS, DMFS, and LRFS, and group differences in these end points were compared by log-rank test. Mean, standard deviation, and range were computed for age. Count (\%) was calculated for all other variables. A two-sided $P<0.05$ was considered as statistically significant. All statistics were twosided, and analyses were performed using SPSS version 22 (IBM Corporation, Armonk, NY, USA).

\section{Results}

\section{Patient demographics and baseline characteristics}

A total of 88 patients were enrolled from November 1, 2005 to December 1, 2009 and randomly assigned to one of the two study arms ( $\mathrm{n}=44$ per arm). Baseline demographics and disease characteristics were similar between the TC and FC treatment groups (Table 1). In both treatment groups, the mean age was $\sim 45$ years, and $\sim 76 \%$ were male. Most patients in both groups had stage III $(58.0 \%)$, T3-T4 (83.0\%), and N1-N2 (69.3\%) cancer.

\section{Treatment completion}

Almost all patients in both arms completed the scheduled two cycles of chemotherapy except one patient in the FC group who withdrew from chemotherapy during cycle 2 (Table 2). Of the original 88 patients, $83 \%$ completed cycle 3 , and $70.5 \%$ completed cycle 4 . A similar percentage of patients completed chemotherapy in both groups $(P \geq 0.161)$.

\section{Toxicity}

No severe adverse events (AEs) occurred during the study (Table 3). During induction therapy, eight grade 3-4 leukopenia (five in the TC group and three in the FC group), ten grade 3-4 neutropenia (six in the TC group and four in the FC group), and one grade 3-4 thrombocytopenia (in the FC group) event were reported. The number of grade 3-4 
Table I Baseline characteristics of 88 patients* receiving TC or FC

\begin{tabular}{lll}
\hline Characteristics & $\begin{array}{l}\text { TC group } \\
(\mathbf{n}=\mathbf{4 4})\end{array}$ & $\begin{array}{l}\text { FC group } \\
\mathbf{( n = 4 4 )}\end{array}$ \\
\hline $\begin{array}{l}\text { Age (years) } \\
\text { Mean (standard deviation) }\end{array}$ & $45.3(8.4)$ & $44.6(8.9)$ \\
Range & $25-60$ & $29-60$ \\
Sex, $\mathbf{n} \%$ & & \\
Male & $34(77.3)$ & $33(75.0)$ \\
Female & $10(22.7)$ & $11(25.0)$ \\
Stage, $\mathbf{n} \%$ & & \\
III & $25(56.8)$ & $26(59.1)$ \\
IVa & $12(27.3)$ & $13(29.5)$ \\
IVb & $7(15.9)$ & $5(11.4)$ \\
T stage, $\mathbf{n} \%$ & & \\
TI & $5(11.4)$ & $0(0)$ \\
T2 & $4(9.1)$ & $6(13.6)$ \\
T3 & $23(52.3)$ & $27(6 I .4)$ \\
T4 & $12(27.2)$ & $11(25.0)$ \\
N stage, $\mathbf{n} \%$ & & $10(22.7)$ \\
N0 & $5(11.4)$ & $15(34.1)$ \\
NI & $16(36.4)$ & $14(3 I .8)$ \\
N2 & $16(36.4)$ & $5(I I .4)$ \\
N3 & $7(15.8)$ &
\end{tabular}

Note: *All patients were at stage M0.

Abbreviations: FC, 5-fluorouracil plus carboplatin; TC, docetaxel plus carboplatin.

AEs increased during chemoradiotherapy. The types and frequency of AEs that occurred during chemoradiotherapy differed between groups. Of grade 3-4 AEs, the most common in the TC group were leukopenia (61.4\%), neutropenia (56.8\%), and alopecia (52.3\%), followed by mucositis (31.8\%) and thrombocytopenia (2.3\%). In the FC group, mucositis (45.5\%) was the most common grade 3-4 AE, followed by leucopenia (25\%), neutropenia (25\%), and thrombocytopenia $(11.4 \%)$. The difference between groups in some of the AEs was significant. A greater percentage of patients treated with FC had leukopenia $(P=0.002)$, neutropenia $(P=0.004)$, and alopecia $(P=0.002)$ of grade $3-4$ compared with the TC group. A higher percentage of patients in the TC group had grade 3-4 thrombocytopenia $(P=0.04)$. A lower percentage of patients in the TC group than in the FC group had nausea ( $52.3 \%$ vs $77.3 \%$, respectively), emesis $(20.5 \%$ vs $45.5 \%$, respectively), diarrhea ( $0 \%$ vs $15.9 \%$, respectively), and

Table 2 Number of patients completing chemotherapy

\begin{tabular}{llll}
\hline Chemotherapy cycles & TC $(\mathbf{n}=\mathbf{4 4})$ & FC $(\mathbf{n}=\mathbf{4 4})$ & P-value \\
\hline Cycle I & $44(100.0)$ & $44(100.0)$ & NA \\
Cycle 2 & $44(100.0)$ & $43(97.7)$ & 0.999 \\
Cycle 3 & $38(86.4)$ & $35(79.5)$ & 0.395 \\
Cycle 4 & $34(77.3)$ & $28(63.6)$ & 0.161 \\
\hline
\end{tabular}

Note: Data are presented as $n(\%)$ and were tested by chi-square test.

Abbreviations: FC, 5-fluorouracil plus carboplatin; NA, not applicable; TC, docetaxel plus carboplatin. mucositis ( $0 \%$ vs $20.5 \%$, respectively) of grade $1-2$ during the induction therapy, but significantly higher proportion of grade $1-2$ alopecia was found in the TC group (100\% in the TC group and $4.5 \%$ in the FC group).

\section{Efficacy}

No difference in treatment response between the two arms was found for cancer present at the nasopharynx and/or lymph node (as defined by the Response Evaluation Criteria in Solid Tumors 1.1) ${ }^{14}(P \geq 0.222)$ (Table 4). After the induction therapy, for cancer present in the nasopharynx or lymph nodes, most patients in the TC and FC groups achieved PR, and the PR was higher for nasopharyngeal cancer than lymph node cancer $(95.5 \%$ and $97.7 \%$ for nasopharyngeal cancer and $71.8 \%$ and $70.6 \%$ for the lymph nodes in the TC and FC groups, respectively). In the TC group, $4.5 \%$ of patients achieved SD for nasopharyngeal cancer, and $12.8 \%$ and $15.4 \%$ achieved CR and SD, respectively, for lymph node cancer. In the FC group, $2.3 \%$ of patients achieved CR for nasopharyngeal cancer, and $23.5 \%$ and $5.9 \%$ achieved CR and SD, respectively, for lymph node cancer. No patients had SD for nasopharyngeal cancer. When combining the findings for both the nasopharynx and lymph nodes, most patients had a partial response $(95.5 \%$ for TC and $97.7 \%$ for FC), and there was no difference between groups.

After the comprehensive (inductive and chemoradiation therapy) treatment, the majority of patients had CR for the combination of nasopharyngeal and lymph node cancers with both TC (81.8\%) and FC (84.1\%) treatment. In both groups, a greater percentage of patients had CR for nasopharyngeal cancer (93.2\% for TC and $90.9 \%$ for FC) than for lymph node cancer $(87.2 \%$ for TC and $85.3 \%$ for FC).

\section{Survival analysis}

At the end of the trial, of the entire study population, 31 patients died, 32 had disease progression, eleven had cancer recurrence, and 25 had distant metastasis. KaplanMeier curves for PFS, OS, DMFS, and LRFS are presented in Figure 1. The PFS rate in the FC group decreased over the follow-up period (86.4\% at Year 1, 70.5\% at Year 3, 65.9\% at Year 5, and $63.6 \%$ at Year 8 [end of the trial]) (Figure 1A). The decrease in rate of PFS was higher at the beginning of the study for the TC group (88.6\% at Year 1 and $63.6 \%$ at Year 3) compared with the FC group but remained stable thereafter such that but both groups had similar rate of PFS at the end of the study. Both FC and TC groups had similar rate of OS, DMFS, DFS, and LRFS. The OS rate in the FC and TC groups was, respectively, $95.5 \%$ and $100 \%$ at Year 1 
Table 3 Drug-related toxicity according to treatments groups

\begin{tabular}{|c|c|c|c|c|c|c|c|}
\hline \multirow[t]{2}{*}{ Therapy } & \multicolumn{3}{|c|}{ TC group $(n=44)$} & \multicolumn{3}{|c|}{ FC group $(n=44)$} & \multirow[t]{2}{*}{$P$-valu } \\
\hline & G0 & GI-2 & G3-4 & G0 & GI-2 & G3-4 & \\
\hline \multicolumn{8}{|c|}{ During induction therapy } \\
\hline Leukopenia & $25(56.8)$ & 14 (3|.8) & $5(11.4)$ & $30(68.2)$ & II (25.0) & $3(6.8)$ & 0.518 \\
\hline Neutropenia & $26(59.1)$ & $12(27.3)$ & $6(13.6)$ & $27(61.4)$ & $13(29.5)$ & $4(9.1)$ & 0.795 \\
\hline Hypochromia & $29(65.9)$ & $15(34.1)$ & $0(0)$ & $32(72.7)$ & $12(27.3)$ & $0(0)$ & 0.488 \\
\hline Thrombocytopenia & $43(97.7)$ & I (2.3) & $0(0)$ & $40(90.9)$ & $3(6.8)$ & I (2.3) & 0.131 \\
\hline Nausea & $21(47.7)$ & $23(52.3)$ & $0(0)$ & $10(22.7)$ & $34(77.3)$ & $0(0)$ & 0.015 \\
\hline Emesis & $35(79.5)$ & $9(20.5)$ & $0(0)$ & $24(54.5)$ & $20(45.5)$ & $0(0)$ & 0.048 \\
\hline Diarrhea & $44(100.0)$ & $0(0)$ & $0(0)$ & $37(84.1)$ & $7(15.9)$ & $0(0)$ & 0.022 \\
\hline Mucositis & $44(100.0)$ & $0(0)$ & $0(0)$ & $35(79.5)$ & $9(20.5)$ & $0(0)$ & 0.007 \\
\hline Liver dysfunction & $37(84.1)$ & $7(15.9)$ & $0(0)$ & $38(86.4)$ & $6(13.6)$ & $0(0)$ & 0.949 \\
\hline Renal dysfunction & $39(88.6)$ & $5(11.4)$ & $0(0)$ & $40(90.9)$ & $4(9.1)$ & $0(0)$ & 0.725 \\
\hline Alopecia & $0(0)$ & $44(100)$ & $0(0)$ & $42(95.5)$ & $2(4.5)$ & $0(0)$ & $<0.001$ \\
\hline \multicolumn{8}{|c|}{ During radiotherapy and chemotherapy } \\
\hline Leukopenia & $5(I I .4)$ & $12(27.3)$ & $27(61.4)$ & $8(18.2)$ & $25(56.8)$ & II (25.0) & 0.002 \\
\hline Neutropenia & $9(20.5)$ & $10(22.7)$ & $25(56.8)$ & $9(20.5)$ & $24(54.5)$ & II (25.0) & 0.004 \\
\hline Thrombocytopenia & $38(86.3)$ & $5(11.4)$ & $I(2.3)$ & $28(63.6)$ & II (25.0) & $5(I I .4)$ & 0.040 \\
\hline Hypochromia & $13(29.5)$ & 31 (70.5) & $0(0)$ & $19(43.2)$ & $25(56.8)$ & $0(0)$ & 0.275 \\
\hline Nausea & $26(59.1)$ & $18(40.9)$ & $0(0)$ & $24(54.5)$ & $20(45.5)$ & $0(0)$ & 0.556 \\
\hline Emesis & $42(95.5)$ & $2(4.5)$ & $0(0)$ & $40(90.9)$ & $4(9.1)$ & $0(0)$ & 0.327 \\
\hline Mucositis & $0(0)$ & $30(68.2)$ & $14(31.8)$ & $0(0)$ & $24(54.5)$ & $20(45.5)$ & 0.274 \\
\hline Liver dysfunction & 34 (77.3) & $10(22.7)$ & $0(0)$ & $35(79.5)$ & $9(20.5)$ & $0(0)$ & 0.539 \\
\hline Renal dysfunction & $36(81.8)$ & $8(18.2)$ & $0(0)$ & $37(84.1)$ & $7(15.9)$ & $0(0)$ & 0.777 \\
\hline Alopecia & $0(0)$ & $21(47.7)$ & $23(52.3)$ & $8(18.2)$ & $36(81.8)$ & $0(0)$ & 0.002 \\
\hline
\end{tabular}

Notes: Data are shown as $\mathrm{n}(\%)$ and were tested by chi-square test or Fisher's exact test. $P$-values shown in bold are significant $(P<0.05)$.

Abbreviations: FC, 5-fluorouracil plus carboplatin; G, grade; TC, docetaxel plus carboplatin.

$81.8 \%$ and $72.5 \%$ at Year 3, $70.5 \%$ and $65.9 \%$ at Year 5, and $65.9 \%$ and $63.5 \%$ at the end of the trial (Figure 1B).

The rate of DMFS in the FC group was $95.5 \%$ and $72.7 \%$ after 1 and 3 years following treatment, respectively, and remained at $70.2 \%$ for another 5.7 years. In the TC group, the DMFS rate was $93.1 \%$ after 1 year of treatment, then dropped to $71.4 \%$ at 30 months following treatment, and remained relatively stable thereafter (Figure 1C). All events of local recurrence were reported within 4 years following treatment. The rates of 1-year, 3-year, and final LRFS were $97.7 \%, 92.6 \%$, and $84.2 \%$ in the FC group and $95.5 \%, 87.8 \%$, and $87.8 \%$ in the TC group, respectively (Figure 1D).

Table 4 Best response

\begin{tabular}{|c|c|c|c|c|c|}
\hline \multirow[t]{2}{*}{ Response } & \multicolumn{2}{|l|}{ TC group } & \multicolumn{2}{|l|}{ FC group } & \multirow[t]{2}{*}{$P$-value } \\
\hline & NP $(n=44)$ & LN (n=39†) & NP $(n=44)$ & $\operatorname{LN}\left(n=34^{\dagger}\right)$ & \\
\hline After induction therapy & & & & & $0.222^{\ddagger} / 0.263^{\S}$ \\
\hline Complete response & $0(0)$ & $5(12.8)$ & I (2.3) & $8(23.5)$ & \\
\hline Partial response & $42(95.5)$ & $28(7 \mid .8)$ & $43(97.7)$ & $24(70.6)$ & \\
\hline Stable disease & $2(4.5)$ & $6(15.4)$ & $0(0)$ & $2(5.9)$ & \\
\hline Combined response $(\mathrm{NP}+\mathrm{LN})$ & & & & & 0.616 \\
\hline Complete response & $0(0.0)$ & & $\mathrm{I}(2.3)$ & & \\
\hline Partial response & $42(95.5)$ & & $43(97.7)$ & & \\
\hline Stable disease & $2(4.5)$ & & $0(0.0)$ & & \\
\hline After comprehensive treatment & & & & & $0.694^{\ddagger} / 0.815^{\S}$ \\
\hline Complete response & $41(93.2)$ & $34(87.2)$ & $40(90.9)$ & $29(85.3)$ & \\
\hline Partial response & $3(6.8)$ & $5(12.8)$ & $4(9.1)$ & $5(\mid 4.7)$ & \\
\hline Combined response $(\mathrm{NP}+\mathrm{LN})$ & & & & & 0.777 \\
\hline Complete response & $36(81.8)$ & & $37(84.1)$ & & \\
\hline Partial response & $8(18.2)$ & & 7 (I5.9) & & \\
\hline
\end{tabular}

Notes: Data are shown as $\mathrm{n}(\%)$ and were tested by chi-square test or Fisher's exact test. ${ }^{\dagger}$ Data on response to LN from 15 patients with stage $\mathrm{N} 0$ were unavailable. ${ }^{\ddagger} P$-value for response rate of NP. ${ }^{\S} P$-value for response rate of $L N$.

Abbreviations: FC, 5-fluorouracil plus carboplatin; LN, lymph node; NP, nasopharynx; TC, docetaxel plus carboplatin. 

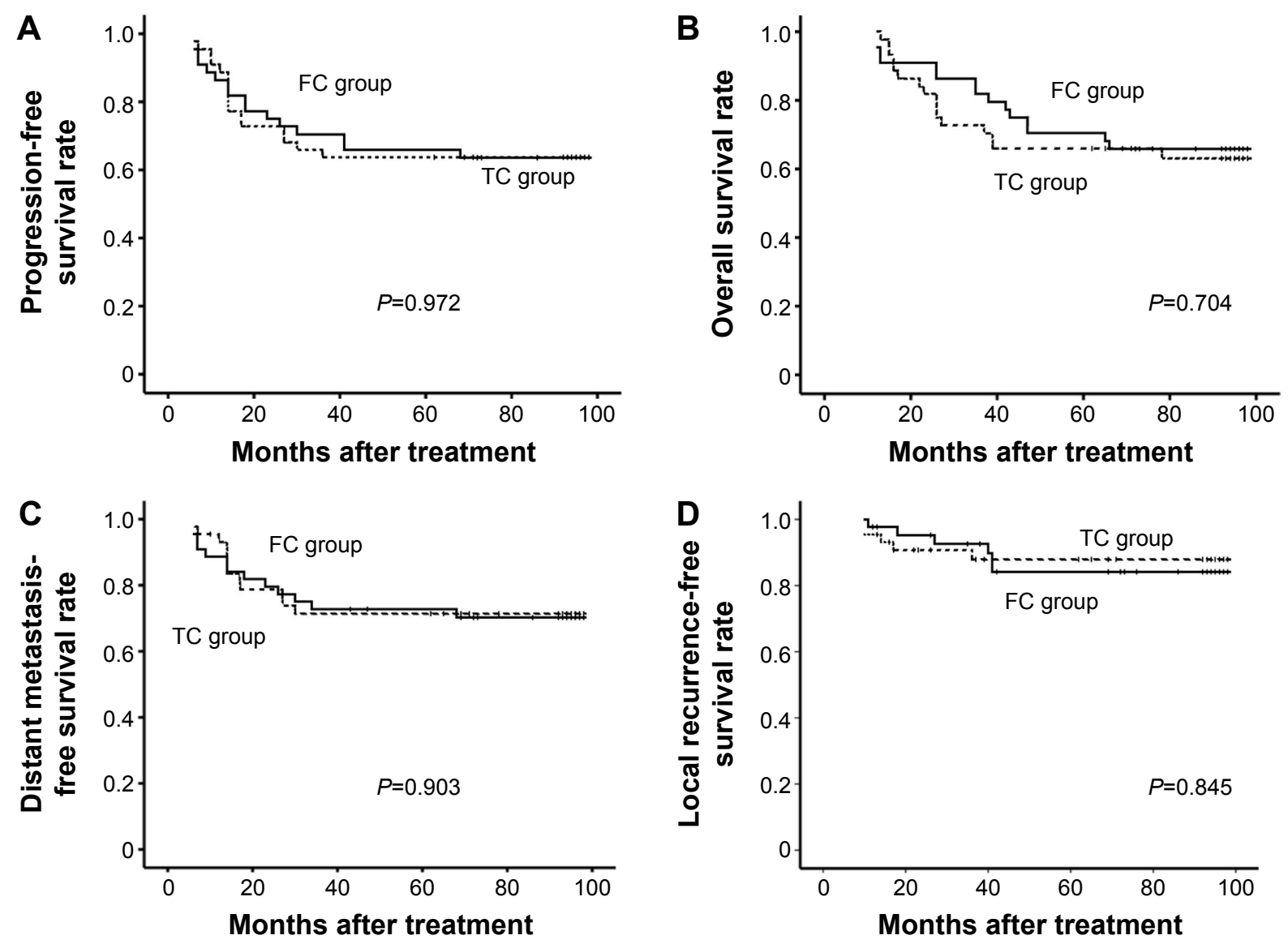

Figure I Kaplan-Meier curves.

Notes: (A) Progression-free survival. (B) Overall survival. (C) Distant metastasis-free survival. (D) Local recurrence-free survival. Abbreviations: FC, 5-fluorouracil plus carboplatin; TC, docetaxel plus carboplatin.

\section{Discussion}

Platinum-based chemotherapy in combination with radiotherapy is a standard treatment strategy for locoregionally advanced NPC., ${ }^{4-17}$ In the present study, the long-term efficacy and tolerability of induction chemotherapy with TC or FC followed by concurrent chemoradiation therapy were evaluated. At the end of the trial, 31 patients died, 32 had disease progression, eleven had cancer recurrence, and 25 had distant metastasis. Overall, there was no difference between treatment groups with regard to response or survival outcomes. We found that following induction and comprehensive therapy, the majority of patients in both treatment groups had CR ( $96 \%-98 \%$ for induction therapy and $82 \%-84 \%$ for comprehensive therapy). PFS, OS, DMFS, and LRFS were also similar between groups. The rate of PFS was $63.6 \%$ for both FC and TC and that of OS was $65.9 \%$ and $63.5 \%$, respectively, at the end of the study. The rate of DMFS was $70.2 \%$ and $71.4 \%$ and that of LRFS was $84.2 \%$ and $87.8 \%$ in the FC and TC groups, respectively. The overall incidence of grade 3-4 AEs in the
TC group (20.5\%) was higher than in the FC group (10.7\%). During concurrent chemoradiotherapy, a greater percentage of patients treated with TC had leukopenia $(P=0.002)$, neutropenia $(P=0.004)$, and alopecia $(P=0.002)$ of grade $3-4$ compared with the FC group, and a greater percentage of patients in the FC group had grade 3-4 thrombocytopenia $(P=0.04)$. In general, these data indicate that TC and FC therapies have similar efficacy in treating NPC and both are well tolerated.

One prior study by Calais et al compared the short-term (1-year) efficacy and tolerability of TC and FC inductive therapies followed by concurrent chemoradiotherapy in patients with locally advanced NPC $(\mathrm{N}=58) .{ }^{13}$ Similar to our long-term study, they found that there was no difference between treatment regimens with regard to $\mathrm{CR}$ and PR immediately following inductive therapy and at 3 months. Immediately following inductive therapy, they found that $\sim 95 \%$ of patients treated with TC and FC had PR, and at 3 months after concurrent chemoradiation therapy, $\sim 95 \%$ achieved CR. These values were comparable to ours in that in our study, 
following inductive therapy, the great majority of patients (96\%-98\%) had PR in both treatment groups. Also, similar to our study, both regimens were well tolerated, and they also found a higher incidence of grade 3-4 AEs in the TC group compared with the FC group (72.4\% vs $37.9 \%$ ). They also found that TC was associated with a greater incidence of leukopenia and neutropenia of grade 3-4, and the FC was associated with a higher rate of grade 3-4 thrombocytopenia. In contrast to our findings, they found that FC was also associated with a higher frequency of emesis compared with TC. Differences between studies may be due to the fact our study followed patients for over 8 years.

Our findings with TC therapy are similar to two other studies that evaluated the efficacy and tolerability of neoadjuvant chemotherapy involving docetaxel and cisplatin followed by concurrent chemoradiation therapy. ${ }^{18,19}$ Both studies followed patients for $\sim 3$ years. In the study of Genet et al, patients were treated with docetaxel and cisplatin followed by concurrent cisplatin plus radiation therapy. ${ }^{18}$ They found that after chemotherapy, the rates of CR and PR were $28.3 \%$ and $56.5 \%$, respectively, and following chemotherapy and concurrent radiotherapy, the rates were $91.3 \%$ and $8.7 \%{ }^{17}$ Posner et al compared the efficacy and tolerability of sequential neoadjuvant chemotherapy with docetaxel plus cisplatin followed by concurrent cisplatin-radiotherapy (CRT) and CRT alone. ${ }^{19}$ Hui et $\mathrm{al}^{9}$ found that docetaxel and cisplatin were associated with a high rate of neutropenia (97\%) during neoadjunctive therapy but found no difference in toxicities between the two treatment arms during CRT. ${ }^{19}$ The rate of 3 -year PFS was greater with the neoadjunctive plus radiotherapy treatment arm $(88.2 \%)$ compared with the CRT $\operatorname{arm}(59.6 \%)$, as also was the rate of OS $(94.1 \%$ vs $67.7 \%$, respectively). ${ }^{19}$ Similar to our study, the studies of Zhong et $\mathrm{al}^{20}$ and Hui et $\mathrm{al}^{9}$ found that neutropenia was one of the most common grade 3-4 AEs.

The use of induction chemotherapy followed by concurrent chemotherapy is of great interest. ${ }^{6,20}$ Several studies have assessed the efficacy and safety of the combination of cisplatin, docetaxel, and 5-FU followed by CRT in treating NPC or head and neck cancer. ${ }^{20-25}$ Overall, the studies found that inductive chemotherapy followed by concurrent chemoradiation was well tolerated and produced clinical benefits in patients with locally advanced NPC.

\section{Limitations}

The findings of this study are limited by the sample size. In addition, the study was conducted in a single site and included only Chinese patients. Hence, it is unclear how generalizable the findings are, particularly to different ethnic groups. Larger, multinational studies are necessary to further explore the use of TC inductive therapy followed by concurrent chemoradiation therapy in patients with locally advanced NPC.

\section{Conclusion}

Our findings indicate that inductive platinum-based chemotherapy followed by concurrent radiotherapy may show survival benefit in patients with locally advanced NPC, although findings from this Phase II study are preliminary. We also found that there was no difference in efficacy or tolerability between treatment regimens containing docetaxel and 5-FU. Large, randomized, multicenter studies are needed to further explore the optimal treatment regimen for patients with NPC.

\section{Acknowledgments}

This study was supported by the National Natural Science Foundation of China (81172041 and 81472525) and National Natural Science Funds for Young Scholar (81201630).

\section{Disclosure}

The authors report no conflicts of interest in this work.

\section{References}

1. Al-Sarraf M, LeBlanc M, Giri PG, et al. Chemoradiotherapy versus radiotherapy in patients with advanced nasopharyngeal cancer: phase III randomized Intergroup study 0099. J Clin Oncol. 1998;16(4): 1310-1317.

2. Kwong DL, Sham JS, Au GK, et al. Concurrent and adjuvant chemotherapy for nasopharyngeal carcinoma: a factorial study. J Clin Oncol. 2004;22(1):2643-2653.

3. Ogawa T, Terada A, Hyodo I, et al. [A Phase I study of docetaxel and cisplatin for advanced squamous cell carcinoma of the head and neck]. Gan To Kagaku Ryoho. 2005;32(7):977-981. Japanese.

4. Chan AT, Ma BB, Lo YM, et al. Phase II study of neoadjuvant carboplatin and paclitaxel followed by radiotherapy and concurrent cisplatin in patients with locoregionally advanced nasopharyngeal carcinoma: therapeutic monitoring with plasma Epstein-Barr virus DNA. J Clin Oncol. 2004;22(15):3053-3060.

5. Baujat B, Audry H, Bourhis J, et al. Chemotherapy in locally advanced nasopharyngeal carcinoma: an individual patient data meta-analysis of eight randomized trials and 1753 patients. Int J Radiat Oncol Biol Phys. 2006;64(1):47-56.

6. Langendijk JA, Leemans CR, Buter J, Berkhof J, Slotman BJ. The additional value of chemotherapy to radiotherapy in locally advanced nasopharyngeal carcinoma: a meta-analysis of the published literature. J Clin Oncol. 2004;22(22):4604-4612.

7. Nabell L, Spencer S. Docetaxel with concurrent radiotherapy in head and neck cancer. Semin Oncol. 2003;30(6 Suppl 18):89-93.

8. Hehr T, Classen J, Belka C, et al. Reirradiation alternating with docetaxel and cisplatin in inoperable recurrence of head-and-neck cancer: a prospective phase I/II trial. Int J Radiat Oncol Biol Phys. 2005;61(5): 1423-1431.

9. Hui EP, Ma BB, Leung SF, et al. Randomized phase II trial of concurrent cisplatin-radiotherapy with or without neoadjuvant docetaxel and cisplatin in advanced nasopharyngeal carcinoma. J Clin Oncol. 2009; 27(2):242-249. 
10. Douple EB, Richmond RC, O’Hara JA, Coughlin CT. Carboplatin as a potentiator of radiation therapy. Cancer Treat Rev. 1985;12 Suppl A: 111-124.

11. Coughlin CT, Richmond RC. Biologic and clinical developments of cisplatin combined with radiation: concepts, utility, projections for new trials, and the emergence of carboplatin. Semin Oncol. 1989; 16(4 Suppl 6):31-43.

12. Calais G, Alfonsi M, Bardet E, et al. Randomized trial of radiation therapy versus concomitant chemotherapy and radiation therapy for advanced-stage oropharynx carcinoma. J Natl Cancer Inst. 1999;91(24): 2081-2086.

13. Calais G, Le Floch O. Radiothérapie et chimiothérapie concomitantes comme traitement des cancers des voies aérodigestives supérieures [Concomitant radiotherapy and chemotherapy in the treatment of cancer of the upper respiratory and digestive tracts]. Bull Cancer Radiother. 1996;83(4):321-329. French.

14. Eisenhauer EA, Therasse P, Bogaerts J, et al. New response evaluation criteria in solid tumours: revised RECIST guideline (version 1.1). Eur J Cancer. 2009;5(2):228-247.

15. Muggia FM. Overview of carboplatin: replacing complementing and extending the therapeutic horizons of cisplatin. Semin Oncol. 1989; 16(2 Suppl 5):7-13.

16. Chitapanarux I, Lorvidhaya V, Kamnerdsupaphon P, et al. Chemoradiation comparing cisplatin versus carboplatin in locally advanced nasopharyngeal cancer: randomised, non-inferiority, open trial. Eur J Cancer. 2007;43(9):1399-1406.

17. Tsukuda M, Mikami Y, Tanigaki Y, et al. Phase I trial of combined chemotherapy with docetaxel, cisplatin, and 5-fluorouracil for patients with locally advanced squamous cell carcinoma of the head and neck. Int J Clin Oncol. 2004;9(3):161-166.

18. Genet D, Cupissol D, Calais G, et al. Docetaxel plus 5-fluorouracil in locally recurrent and/or metastatic squamous cell carcinoma of the head and neck: a phase II multicenter study. Am J Clin Oncol. 2004;27(5): $472-476$.
19. Posner MR, Hershock DM, Blajman CR, et al. Cisplatin and fluorouracil alone or with docetaxel in head and neck cancer. $N$ Engl J Med. 2007;357(17):1705-1715.

20. Zhong YH, Dai J, Wang XY, et al. Phase II trial of neoadjuvant docetaxel and cisplatin followed by intensity-modulated radiotherapy with concurrent cisplatin in locally advanced nasopharyngeal carcinoma. Cancer Chemother Pharmacol. 2013;71(6):1577-1583.

21. Lee AW, Ngan RK, Tung SY, et al. Preliminary results of trial NPC0501 evaluating the therapeutic gain by changing from concurrentadjuvant to induction-concurrent chemoradiotherapy, changing from fluorouracil to capecitabine, and changing from conventional to accelerated radiotherapy fractionation in patients with locoregionally advanced nasopharyngeal carcinoma. Cancer. 2015;121(8):1328-1338.

22. Kong L, Hu C, Niu X, et al. Neoadjuvant chemotherapy followed by concurrent chemoradiation for locoregionally advanced nasopharyngeal carcinoma: interim results from 2 prospective phase 2 clinical trials. Cancer. 2013;119(23):4111-4118.

23. Huang PY, Zeng Q, Cao KJ, et al. Ten-year outcomes of a randomised trial for locoregionally advanced nasopharyngeal carcinoma: a singleinstitution experience from an endemic area. Eur J Cancer. 2015;51(13): 1760-1770.

24. Johnson FM, Garden A, Palmer JL, et al. A Phase II study of docetaxel and carboplatin as neoadjuvant therapy for nasopharyngeal carcinoma with early T status and advanced N status. Cancer. 2004;100(5): 991-998.

25. Bae WK, Hwang JE, Shim HJ, et al. Phase II study of docetaxel, cisplatin, and 5-FU induction chemotherapy followed by chemoradiotherapy in locoregionally advanced nasopharyngeal cancer. Cancer Chemother Pharmacol. 2010;65(3):589-595. 


\section{Supplementary materials}

Table SI Neck region requiring prophylactic irradiation

\begin{tabular}{ll}
\hline Neck region with metastatic lymph nodes & Neck region requiring prophylactic irradiation (CTV2 in the neck) \\
\hline No & Bilateral II, III, Va \\
II & Ipisilateral II, III, IV, Va, Vb \\
III & Ipisilateral II, III, IV, Va, Vb \\
Va & Ipisilateral II, III, IV, Va, Vb \\
IV & Ipisilateral II, III, IV, Va, Vb, supraclavicular region \\
Vb & Ipisilateral II, III, IV, Va, Vb, supraclavicular region \\
Unilateral neck & Bilateral II, III, Va
\end{tabular}

Notes: III region is included for CTV2 when the following conditions are present: (I) Va region with metastatic lymph nodes is $\geq 3 \mathrm{~cm}$; (2) IV region with metastatic lymph nodes has extracapsular involvement; (3) Vb region shows the pushing of the submandibular gland by metastatic lymph nodes or unclear borderline with the submandibular gland; and (4) several regions (four or more) show lymph node metastasis.

Abbreviation: CTV2, clinical target volume 2 .

Table S2 Dose adjustment of chemotherapy due to adverse events as defined by the National Cancer Institute Common Terminology Criteria for Adverse Events 3.0

\begin{tabular}{ll}
\hline Serious AE & Dose adjustment \\
\hline $\begin{array}{l}\text { Homological toxicity } \\
\text { Fever due to agranulocytosis }\end{array}$ & $\begin{array}{l}75 \% \text { of standard dose } \\
\text { Neutropenia (grade } 3-4 \text {, temperature } \geq 38.5^{\circ} \mathrm{C} \text {, or with concomitant bleeding) }\end{array}$ \\
$\begin{array}{l}\text { Gastrointestinal toxicity } \\
\text { Vomiting/nausea (grade } 3 \text { following preventive or symptomatic treatment) }\end{array}$ & $\begin{array}{l}75 \% \text { of standard dose } \\
\text { Vomiting/nausea (grade } 3 \text { following preventive or symptomatic treatment) }\end{array}$ \\
$\begin{array}{l}\text { Kidney } \\
\text { Creatinine clearance }- \text { calculated value } 35-49 \mathrm{~mL} / \mathrm{min}\end{array}$ & $\begin{array}{l}50 \% \text { of standard dose } \\
\text { chemotherapy was } 2 \text { weeks }\end{array}$ \\
$\begin{array}{l}\text { Creatinine clearance }- \text { measured value } 45-59 \mathrm{~mL} / \mathrm{min} \\
\text { Liver }\end{array}$ & Discontinuation of chemotherapy if toxicity was not resolved \\
Grade 3 toxicity & No adjustment \\
Grade 4 toxicity & Discontinue chemotherapy
\end{tabular}

Notes: Data from the website of the National Cancer Institute (http://www.cancer.gov). Common Terminology Criteria for Adverse Events v3.0 (CTCAE); published August 9, 2006. Available from: http://ctep.cancer.gov/protocolDevelopment/electronic_applications/docs/ctcaev3.pdf. Accessed July 6, 2016.'

Abbreviation: $\mathrm{AE}$, adverse event.

\section{Reference}

1. National Cancer Institute. Common Terminology Criteria for Adverse Events v3.0 (CTCAE); published August 9, 2006. Available from: http:// ctep.cancer.gov/protocolDevelopment/electronic_applications/docs/ ctcaev3.pdf. Accessed July 6, 2016.

\section{Publish your work in this journal}

OncoTargets and Therapy is an international, peer-reviewed, open access journal focusing on the pathological basis of all cancers, potential targets for therapy and treatment protocols employed to improve the management of cancer patients. The journal also focuses on the impact of management programs and new therapeutic agents and protocols on

\section{Dovepress}

patient perspectives such as quality of life, adherence and satisfaction. The manuscript management system is completely online and includes a very quick and fair peer-review system, which is all easy to use. Visit http://www.dovepress.com/testimonials.php to read real quotes from published authors. 